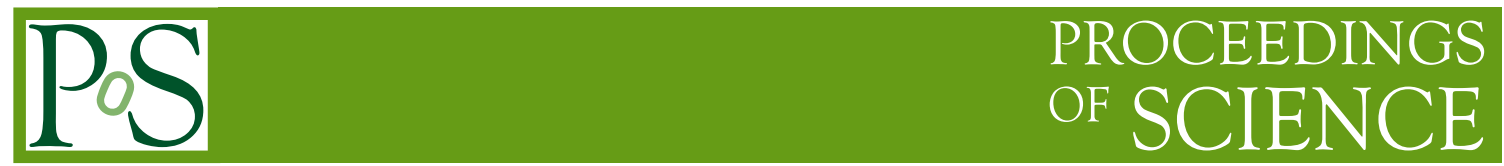

\title{
An anomalous dust emission component? The Dust processes
}

\author{
Laurent Verstraete* and Nathalie Ysard \\ Institut d'Astrophysique Spatiale, UMR8617, Université Paris-Sud, F-91405, Orsay,France \\ E-mail: laurent.verstraetedias.u-psud.fr
}

\begin{abstract}
Measurements of CMB fluctuations will be performed most sensitively between 1 and $100 \mathrm{GHz}$ (Banday et al. 2003, Fig. 6). In this frequency range however, several foregrounds limit the access to the CMB. Among these, the so-called anomalous emission which is probably of dust origin and is the least well known, motivating further theoretical and observational studies. A better understanding of this emission will help remove galactic foregrounds and will bring new constraints on interstellar grains. We discuss here several dust emission processes that could be at the origin of this anomalous emission. Emphasis is given to spinning, small dust grains which seem to be favored by recent data analysis.
\end{abstract}

$C M B$ and Physics of the Early Universe

20-22 April 2006

Ischia, Italy

\footnotetext{
* Speaker.
} 


\section{A dust origin to the anomalous emission}

Since the discovery of an unexpected (anomalous) emission in the 1-100 GHz range (Kogut et al. 1996), clear evidence came along that this foreground is related to dust as demonstrated in this volume by R. Davies. In particular, this microwave emission appears more correlated to near- and mid-IR dust emission (as traced by the 12 and $25 \mu \mathrm{m}$ IRAS bands, Casassus et al. 2006) and seems to disappear in the vicinity of hot stars (Dickinson et al. 2006). Moreover, the degree of linear polarization of this anomalous emission appears to be low (less than 5\%, Battistelli et al. 2006).

In this frequency range, several mechanisms involving dust at low-energy, low-temperature may produce detectable emission. The first to be proposed was emission from very small, rotating interstellar grains (Draine \& Lazarian 1998) ${ }^{1}$. Shortly after, the same authors showed that magnetization fluctuations of big grains (1000 $\mathrm{nm}$ in radius) may also produce a significant emission at $\mathrm{GHz}$ frequencies. In the following sections we briefly review the main features of these emission mechanisms and highlight their observational consequences.

\section{Microwave emission of small, spinning dust grains}

>From IR spectroscopy, it is known that small, sub-nanometric interstellar grains are aromatic molecules or PAHs (Polycyclic Aromatic Hydrocarbons, Léger \& Puget 1984, Allamandola et al. 1985) containing a few 10 to 100 carbon atoms (a few $\AA$ in radius). In the general interstellar medium, PAHs can absorb stellar photons whereby they are raised to temperatures of a few 100 $\mathrm{K}$. They then cool off by rovibrational emission in characteristic IR-bands between 3 and $17 \mu \mathrm{m}$. PAHs therefore emit during temperature fluctuations (see Fig. 2 in Ponthieu \& Martin, this volume). PAHs can also undergo collisions with gas phase species. As a result of these processes, PAHs are likely charged and in radicalar form (Le Page et al. 2003). In particular radical, charged PAHs may carry a significant permanent electric dipole moment (a few tenths of Debye). Due to the above frequent and random excitations, PAHs are spinning in space and will produce electric dipole emission from rotational levels.

The rotation of PAHs in space was first studied by Rouan et al. $(1992,1997)$ motivated by the further development of the PAH hypothesis and its observable consequences, e.g., the possible polarization of PAH IR emission bands and the rotational broadening of electronic transitions in PAHs and its relevance for the interpretation of diffuse interstellar absorption bands. In 1998, Draine \& Lazarian took another view at this problem with particular emphasis on gas-grains interactions and gave the first estimate of the rotational emission spectrum in the 1-100 $\mathrm{GHz}$ range. They showed that the emission of spinning dust indeed peaks in the range 1 to $100 \mathrm{GHz}$ but also found that this emission is sensitive to the physical state of the gas (density, temperature). However, strong assumptions were made in this work motivating further study ${ }^{2}$.

\footnotetext{
${ }^{1}$ Interestingly, this field was pioneered by Erickson (1957) from a different perspective, i.e., that of noise in radio observations.

${ }^{2}$ In particular, it was assumed that the energy of the emitted photon is much smaller than the internal energy (canonical statistics). In the case of small grains that spend most of their time at low energy (Désert et al. 1986, Guhatakurta \& Draine 1989) this hypothesis must be revised.
} 
We therefore develop a new model where PAHs are isolated systems (microcanonical statistics) and treat the rotation motion quantally (Ysard \& Verstraete, in preparation). The dynamics of rotation include all the gas-grain interactions from Draine \& Lazarian (1998) and the IR rovibrational emission that cools off the molecule after absorption of a stellar visible-UV photon. We assume that the vibrational motion is harmonic and that the molecule rotates as a rigid rotor. Based on stability arguments, PAHs are taken to be compact, planar molecules and are treated as symmetric tops for the rotation. We note $C$ the rotational constant for an axis perpendicular to the molecular plane and $B$ the rotational constant for an in-plane axis $(B=2 C)$. The rotational energy is then $E=B\left[J(J+1)-M_{J}^{2} / 2\right]$.

Following the exact statistical method described in Draine \& Li (2001), we first determine the rovibrational density of states using the IR properties of PAHs as recently constrained from ISO and Spitzer data (Rapacioli et al. 2005, Flagey et al. 2006). For each PAH size, we then solve the detailed balance of populations in a given radiation field and obtain the probability distribution $P(E)$ for the molecule to be in the state of internal energy $E$. The rotational excitation is treated as a stationary random walk. Equating the rates of change of $J$ for all processes (gas-grains interactions and IR emission), we solve for the equilibrium value $J_{0}$ of the rotational quantum number . In all environments where stellar photons are present, even in shielded regions (down to visible-UV intensities of a few percents of the standard interstellar radiation field), we find that the rotational excitation by IR emission dominates. In that case, it can be shown that the distribution of the rotational number $J$ for a given molecular size and in a given vibrational level is: $n(J)=n_{0}(2 J+$ $1)^{2} \exp \left(-J^{2} / J_{0}^{2}\right)$. Since the interstellar medium is pervaded by stellar light, this result applies at large scale in the Galaxy. Further on, following Rouan et al. (1992) we assume that $n(J)$ is independent of the vibrational excitation.

The rotational emission spectrum is finally obtained from a sum over the size distribution of PAHs (in terms of the number of carbon atoms $N_{C}$ in the molecule), $n_{s}\left(N_{C}\right)$ with minimum and maximum sizes of $N_{1}$ and $N_{2}$ respectively:

$$
I=\int_{N_{1}}^{N_{2}} A_{J, J-1} n\left(J, N_{C}\right) \frac{2 B J}{2 B c} n_{s}\left(N_{C}\right) d N_{C}
$$

where we ascribe a bandwith of $2 B c$ to each rotational transition. The frequency spectrum is then obtained from the expression of the rotational energy given above. We assume that the electric dipole of a PAH is 0.2 Debye, independent of $\operatorname{size}^{3}$. The size distribution of PAHs is taken to be either a power law $\left(n_{s}\left(N_{C}\right) \sim N_{C}^{-2.25}\right.$ or $n_{s}(a) \sim a^{-3.5}$ in terms of the molecular radius) or a log-normal law as proposed more recently (Weingartner \& Draine 2001) in consistency with stability studies of PAHs in the interstellar medium (Le Page et al. 2003). Both these PAH size distributions are compatible with observational constraints, in particular the dust extinction curve at UV wavelengths.

On Fig. 1, we show that the influence of the size distribution on the rotation emission spectrum is modest. In fact, this spectrum mostly depend on the abundance of the smallest PAHs (containing less than 50 carbon atoms) because they have the highest rotational constant. We also show on Fig. 1 the influence of the radiation field: while its intensity is multiplied by a factor $410^{4}$, the

\footnotetext{
${ }^{3}$ This is a typical value for an unsaturated bond in PAHs (Draine \& Lazarian 1998).
} 
PAH rotation emission is raised by a factor less than 20. This lack of sensitivity to the intensity of the radiation field is due to the fact that the rotational excitation only depends on the rovibrational energy content of the molecule, not on the absorption rate.

The left panel of Fig. 2 presents the result of our model in the case of the Perseus Arm
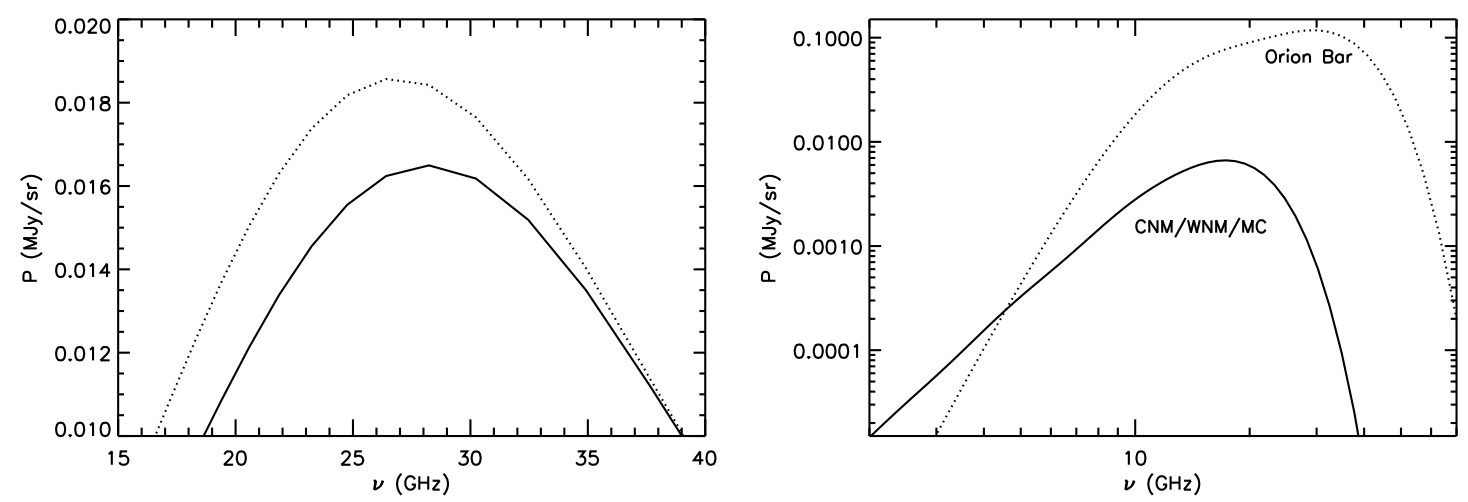

Figure 1: Left panel: Shows the influence of the size distribution on the PAH rotational emission spectrum with a constant PAH abundance (see Fig. 2 caption). The solid line corresponds to a power law size distribution $n_{s}\left(N_{C}\right) \sim N_{C}^{-2.25}$ where $N_{C}$ the number of carbon atoms ranges from 38 to 206 . The dotted line shows the case of a log-normal size distribution of centroid $N_{C}=35$ and $\sigma=0.3$ as in Weingartner \& Draine (2001). Right panel: Shows the influence of the radiation field again with a fixed PAH abundance. The solid line corresponds to the standard interstellar radiation field (Mathis et al. 1984) where the visible-UV part is equivalent to a blackbody of temperature $20,000 \mathrm{~K}$. The dotted line shows the case of the Orion Bar where the excitation is much higher (the UV part of the spectrum amounts to a blackbody at 37,000 $\mathrm{K}$ with an intensity $410^{4}$ higher than in the previous case).
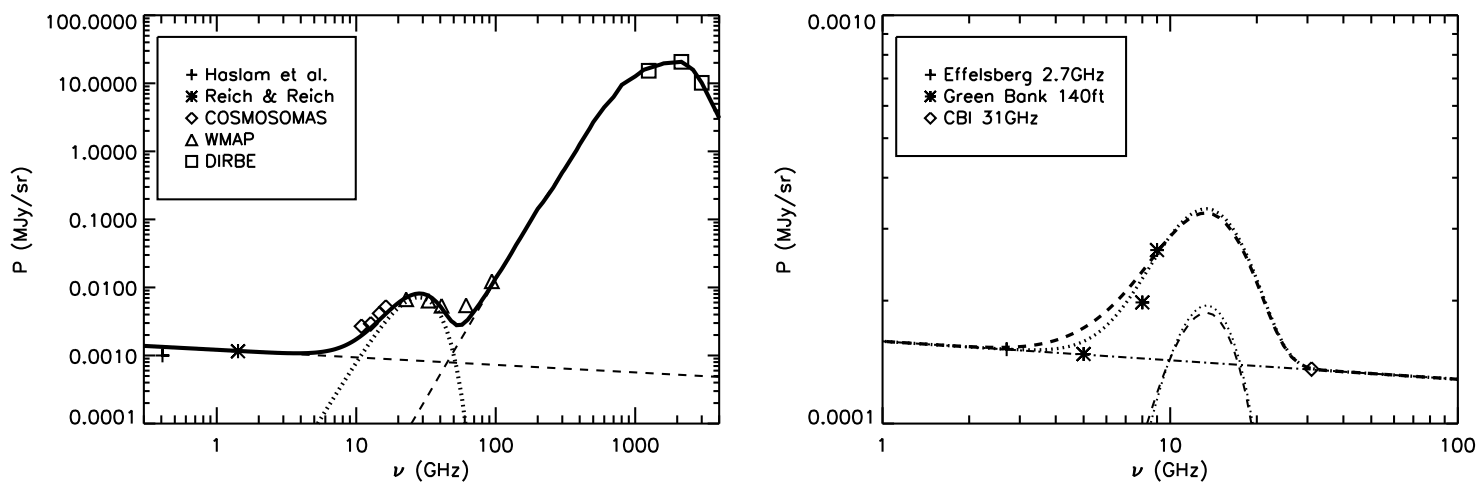

Figure 2: Left Panel: The case of the Perseus Arm (Watson et al. 2005). At low frequency (1-10 GHz), the free-free emission dominates (dashed line) whereas at high frequency $(100-1000 \mathrm{GHz})$, the dust thermal emission is the main contributor (dashed line). The dotted line is the result of our model with a power law size distribution as in Fig. 1. The abundance of carbon in PAHs is $[\mathrm{C} / \mathrm{H}]=1.610^{-5}$ i.e. $6 \%$ of the interstellar carbon. Right Panel: The case of a diffuse HII region (Dickinson et al. 2006). Our model is the dotted line with a PAH abundance of $1 \%$ of interstellar C and a power law size distribution $n_{s}\left(N_{C}\right) \sim N_{C}^{-2.25}$ where $N_{C}$ ranges from 73 to 206. 
environment. Combined to the free-free and thermal dust components given in Watson et al. (2005), a good match is obtained for a PAH abundance of about half that required to explain the 3-17 $\mu \mathrm{m}$ IR bands (see Fig. 1 of Ponthieu \& Martin, this volume) or $6 \%$ of the interstellar carbon available for dust grains. In the case of a diffuse, ionized (HII) region, we require larger PAHs with an abundance 6 times smaller. Such a PAH depletion is expected in regions of high excitation and intense radiation field and is indeed observed (Kassis et al. 2006 and references therein).

First conclusions from this work are, 1) with a more detailed treatment of the physics of PAHs in space, we confirm that the emission of spinning, small PAHs can explain the observed anomalous emission, assuming a reasonable abundance of small PAHs and a plausible dipole moment; 2) as in Rouan et al. (1992), we find that the rotational excitation is dominated by the rovibrational emission cascade and that it is not very sensitive to the intensity of the radiation field: a direct consequence of this is that the emission of spinning PAHs is roughly the same in the variety of environments of the diffuse interstellar medium, in contrast with the findings of Draine \& Lazarian (1998); 3) the emission of spinning PAHs is sensitive to the fraction of small PAHs (less than 100 C), providing an important constraint to models of interstellar PAHs.

\section{Microwave emission from big grains}

Larger grains of sub-micron radius also contribute significantly to the sky emission at frequencies 10 to $100 \mathrm{GHz}$ (see Fig. 2, left panel). Such big grains (BGs), because of their large heat capacity, remain at low ( 10 to $20 \mathrm{~K}$ ) equilibrium temperature $T_{d}$ when heated by stellar photons. The electric dipole emission of BGs can be described by a modified blackbody law $I \sim v^{\beta} B_{v}\left(T_{d}\right)$ and is therefore often called thermal dust emission. BGs are composed of carbonaceous (graphite) and amorphous silicate compponents and models predict that the far-IR to $\mathrm{cm}$ BG emission is dominated by silicates (Li \& Draine 2001).

The emission of BGs probably has a more complex behaviour. Both the structure of the grain (porosity, inhomogeneity) and its energy dissipation channels at low-temperatures play an important role in shaping its $\mathrm{mm}$ to $\mathrm{cm}$-emission. Indeed, recent mm-observations suggest that smaller grains $(1-10 \mathrm{~nm})$ may stick on the surface of big grains thus enhancing their mm-emissivities (Stepnik et al. 2003). On the other hand, there is also observational evidence for an emissivity index $\beta$ that depends on the grain temperature $T_{d}$ (Dupac et al. 2003). A similar temperature behaviour has been observed in the laboratory and may point at the presence of two-level systems (TLS) in silicates (Agladze et al. 1996, Boudet et al. 2005). TLS are well known in the context of glasses and arise from impurities and/or disorder in the bulk solid. These studies show that TLS are important to understand the dust thermal emission and will provide direct information on the structure of BGs. TLS may also be relevant for the anomalous emission. Indeed, the existence of a resonant transition $^{4}$ around $20 \mathrm{GHz}$ in silicates has been reported by Phillips (1987). The spectral shape of the associated emission however requires the knowledge of the density of TLS-states, which is missing. Further laboratory and modelling studies are therefore needed to clarify the contribution of TLS to the emission of BGs: this is one of the goals of a 3-year research program just proposed

\footnotetext{
${ }^{4}$ The 2 levels involved correspond to coupled rotation states of neighbouring $\mathrm{SiO}_{4}$ tetrahedra (Phillips 1987).
} 
to the french research agency by IAS and CESR (Centre d'Etudes Spatiales et du Rayonnement).

On the other hand, Draine \& Lazarian (1999) have pointed out that magnetic dipole emission of BGs may present a broad resonant emission between 10 and $100 \mathrm{GHz}$. To emit at the flux level and frequency position of the observed anomalous component, this mechanism implies a rather large spontaneous magnetization, not too large though so as to keep the gyrofrequency in the right range $(\sim 6 \mathrm{GHz})$ to place the emission peak at 10 to $50 \mathrm{GHz}$. At present no terrestrial analogue of interstellar BGs has such properties. Thus Draine \& Lazarian presented emission predictions for a fiducial material whose properties are intermediate between magnetite and metallic iron and which would include most interstellar iron. The authors concluded that with current knowledge, a magnetic dipole contribution to the anomalous component could not be excluded although less likely than the spinning PAHs explanation. A complete assessment of this hypothesis will await measurements of magnetic susceptibility on interstellar grains analogues in the 1-100 GHz range. New highlight on this topic comes from the recent laboratory results of Davoisne et al. (2006). To understand the evolution of silicates in the interstellar medium (in particular the transition from the crystalline to amorphous phase), these authors have heated olivine samples to $\sim 1000 \mathrm{~K}$ as could occur in a proto-solar nebula. They found that in a carbon-rich atmosphere all the iron of the olivine sample aggregated to form metallic iron nano-particles. This result has important bearing for the alignment of BGs (see Ponthieu \& Martin this volume) and maybe also for the anomalous emission. Measurements of magnetic susceptibility are currently underway to better assess the importance of this result for the emission and polarization of the sky at microwave frequencies.

\section{Summary}

Several dust related mechanisms are plausible explanations for the anomalous emission between 10 and $100 \mathrm{GHz}$. Among these mechanisms, the emission of spinning permanent dipoles of small (a few $\AA$ in radius) hydrocarbons called PAHs appears most promising because of the available evidence. Observations indeed suggest a relationship between the IR tracers of small grains (IRAS $12 \mu \mathrm{m}$-band) and the anomalous emission. This emission also appears as little polarized $(P<5 \%)$ as predicted by alignment models of small dust grains (Lazarian \& Roberge 1997). The field of interstellar PAHs also benefits from numerous laboratory, theoretical, observational and modelling studies over the past two decades in order to understand the IR bands which ubiquitoulsy trace interstellar matter.

We have discussed here the first results of a new model of spinning PAHs emission at GHz frequencies. This model improves on previous studies because it treats PAHs as isolated systems, so as in space, and includes the results of recent IR satellite observations for the rovibrational emission of PAHs. We show that the rotational excitation of small, radicalar PAHs can indeed explain the anomalous emission with a reasonable carbon budget (6\% of the carbon available for dust). We also show that the PAH rotational excitation is not very sensitive to the intensity of the radiation field leading to the prediction that the resulting emission will not change much over the entire sky, as suggested by recent observations of the anomalous emission (Lagache 2003, Davies et al. 2006). 
A closer assessment of the contribution of bigger grains (with radii of a few $1000 \mathrm{~nm}$ ) still awaits more laboratory studies on their properties, in particular silicates, at low-temperature. Such studies are currently underway or will start soon: they are motivated by the understanding of the thermal dust and anomalous emission as well as the alignment properties of interstellar that leads to significant degrees of polarisation at microwave frequencies.

\section{References}

[1] Agladze, N. I.; Sievers, A. J.; Jones, S. A.; Burlitch, J. M.; Beckwith, S. V. W., 1996, ApJ 462, 1026

[2] Allamandola, L.J., Tielens, A.G.G.M, Barker, J.R., 1985, ApJ 290, L25

[3] Banday, A. J.; Dickinson, C.; Davies, R. D.; Davis, R. J.; Gorski, K. M., 2003, MNRAS 345, 897

[4] E.S. Battistelli, R. Rebolo, J.A. Rubió, S.R. Hildebrandt, R.A. Watson, C. Gutiérrez, R.J. Hoyland, 2006, astro-ph 0603379

[5] Boudet, N.; Mutschke, H.; Nayral, C.; Jäger, C.; Bernard, J.-P.; Henning, T.; Meny, C., 2005, ApJ 633,272

[6] Désert, F.-X., Boulanger, F., Shore, S.N., 1986, A\&A 160, 295

[7] Casassus, S.; Cabrera, G. F.; Forster, F.; Pearson, T. J.; Readhead, A. C. S.; Dickinson, C., 2006, ApJ 639,951

[8] Davies, R. D.; Dickinson, C.; Banday, A. J.; Jaffe, T. R.; Gorski, K. M.; Davis, R. J., 2005, astro-ph 11384

[9] Davoisne, C.; Djouadi, Z.; Leroux, H.; D’Hendecourt, L.; Jones, A.; Deboffle, D., 2006, A\&A 448, L1

[10] Dickinson, C.; Casassus, S.; Pineda, J. L.; Pearson, T. J.; Readhead, A. C. S.; Davies, R. D., 2006, ApJ 643, L111

[11] Draine, B.T., Lazarian, A., 1998, ApJ 508, 157

[12] Draine, B.T., Li, A., 2001, ApJ 551, 807

[13] Dupac, X.; Bernard, J.-P.; Boudet, N.; Giard, M.; Lamarre, J.-M.; MŐny, C.; Pajot, F.; Ristorcelli, I.; Serra, G.; Stepnik, B.; Torre, J.-P., 2003, A\&A 404, L11

[14] N. Flagey, F. Boulanger, L. Verstraete, M. A. Miville Deschênes, A. Noriega Crespo and W. T. Reach , 2006, A\&A 453, 969

[15] Kassis, Marc; Adams, Joseph D.; Campbell, Murray F.; Deutsch, Lynne K.; Hora, Joseph L.; Jackson, James M.; Tollestrup, Eric V., 2006, ApJ 637, 823

[16] A. Kogut, A. J. Banday, C. L. Bennett, K. M. Gorski, G. Hinshaw, G. F. Smoot, and E. L. Wright, 1996, ApJ 460, 1

[17] Lagache,G., 2003, A\&A 405, 813

[18] Lazarian, A.; Roberge, W.G., 1997, ApJ 484, 230

[19] Léger, A., Puget, J-L., 1984, A\&A 137, L5

[20] Le Page, V., Snow, T.P., Bierbaum, V.M., 2003, ApJ 316, 330

[21] Phillips, W.A., 1987, Rep. Prog. Phys. 50, 1657 
[22] Mathis, J. S.; Mezger, P. G.; Panagia, N., 1983, A\&A 128, 212

[23] Rapacioli, M.; Joblin, C.; Boissel, P., 2005, A\&A 429, 193

[24] Rouan, D.; Léger, A.; Omont, A.; Giard, M., 1992, A\&A 253, 498

[25] Rouan, D.; Leger, A.; Le Coupanec, P., 1997, A\&A 324, 661

[26] Stepnik, B.; Abergel, A.; Bernard, J.-P.; Boulanger, F.; CambrŐsy, L.; Giard, M.; Jones, A. P.; Lagache, G.; Lamarre, J.-M.; Meny, C.; Pajot, F.; Le Peintre, F.; Ristorcelli, I.; Serra, G.; Torre, J.-P.,2003, A\&A 398, 551

[27] Watson, R. A.; Rebolo, R.; Rubió-Martín, J. A.; Hildebrandt, S.; Gutiérrez, C. M.; Fernández-Cerezo, S.; Hoyland, R. J.; Battistelli, E. S., 2005, ApJ 624, L89

[28] Weingartner, J.C., Draine, B.T., 2001, ApJ 548, 296 